\title{
The dual approach to stationary and evolution quasilinear PDEs
}

\author{
Alessandro Selvitella
}

\begin{abstract}
In this paper, we study several quasilinear PDEs with a particular algebraic structure. In the case of stationary solutions for a quasilinear Schrödinger equation, Colin and Jeanjean (Nonlinear Anal 56:213-226, 2004) implemented a dual approach to prove existence and qualitative properties of the solutions. The method takes advantage of the particular underlying structure of that quasilinear PDE, which is essentially semilinear. The main goal of this manuscript is to show that the dual approach is successful in a broader set of problems, especially in the stationary cases involving more general quasilinear terms and the $p$-Laplacian. We prove also that this approach works for some quasilinear heat equations, but fails for the complete evolution of quasilinear Schrödinger equations. The reason of the failure seems related to the extra structure of the complex plane.
\end{abstract}

Mathematics Subject Classification. 35Q55, 35J60, 35K55.

\section{Introduction}

We consider the following quasilinear Schrödinger equation:

$$
i \partial_{t} \phi(t, x)+\Delta \phi(t, x)+\lambda \phi(t, x) \Delta|\phi(t, x)|^{2}+|\phi(t, x)|^{p-1} \phi(t, x)=0,
$$

where $i$ is the imaginary unit, $N \geq 1$ is the space dimension, $p>1$ is the exponent of the semilinear term, the domain is $(t, x) \in(0, \infty) \times \mathbf{R}^{N}$ and $\phi: \mathbf{R}^{N} \rightarrow \mathbf{C}$ is the wave function. This equation appears in different physical models, such as the superfluid film equation in plasma physics. We refer to [6] and [7], for a more detailed bibliography on the physical background.

The mathematical theory for this equation is more involved than the semilinear case, especially for what concerns the short time dynamics. In the study of the Cauchy problem, the presence of the quasilinear term causes the phenomenon called loss of derivatives. To overcome this problem, mathematicians assume high regularity on the initial datum and prove local well-posedness in Sobolev spaces of high order. Because of this lack of local wellposedness 
in lower regular spaces, a Gagliardo and Nirenberg type inequality [6] cannot guarantee global wellposedness in the energy space. We refer to Colin [4], Colin et al. [6], Kenig et al. [7], Lange et al. [8] and Poppenberg [14,15], Selvitella and Wang [20] for the main results, concerning well-posedness of this problem and the long time behavior of solutions. To our knowledge, the latest results in this area are due to Marzuola et al. in [12] and [13].

We have a better understanding for what concerns the existence of particular types of stationary solutions, called standing waves, namely solutions which model particles at rest in the form $\phi(t, x)=u(x) e^{i \nu t}$. Here, $u: \mathbf{R}^{N} \rightarrow \mathbf{C}$ solves the quasilinear elliptic equation:

$$
-\Delta u-u \Delta|u|^{2}+\nu u-|u|^{p-1} u=0,
$$

Many authors have studied solutions to this problem. See, for example, Liu et al. [9], Liu et al. [10], Liu and Wang [11] and Colin and Jeanjean [5]. The most complete result concerning ground states of (2) is due to Colin et al. [6]. In [6], the authors prove that ground states exist, are real and positive (up to phase shifts) classical solutions, they decay exponentially at infinity with their first and second derivatives (actually with all their derivatives, see Selvitella [18]) and, moreover, they are radial with respect to some point and nondegenerate (see Selvitella [19]).

The method employed by Colin and Jeanjean in [5] and Colin et al. in [6], relies on a magic transformation which sends the original problem to a semilinear one. The method takes advantage of the particular algebraic structure of this quasilinear PDE. The transformation is defined through the following ODE:

$$
\left\{\begin{array}{l}
F^{\prime}(s)=\frac{1}{\sqrt{1+2 F^{2}(s)}} \\
F(0)=0 .
\end{array}\right.
$$

The main goal of this manuscript is to show that this approach is successful in a broader set of problems. We start by considering the equation

$$
-\Delta u-u l^{\prime}\left(|u|^{2}\right) \Delta l\left(|u|^{2}\right)=\omega u-f\left(|u|^{2}\right) u=: g(u) .
$$

For this type of problems, we can prove the following theorem.

Theorem 1.1. Suppose that

- $(\mathrm{H} 0) g(s)$ is locally Hölder continuous in $[0,+\infty)$;

- (H1) for $N \geq 3$

$$
-\infty<\liminf _{s \rightarrow 0} \frac{g(s)}{s} \leq \limsup _{s \rightarrow 0} \frac{g(s)}{s}=-\nu<0,
$$

while for $N=1,2$,

$$
\lim _{s \rightarrow 0} \frac{g(s)}{s}=-\nu<0
$$

- (H2) for $N \geq 3$

$$
\lim _{s \rightarrow+\infty} \frac{g(s)}{\sqrt{1+2 l^{\prime}\left(F^{2}(s)\right) F^{2}(s)} s^{\frac{N+2}{N-2}}}=0,
$$


while for $N=2$, for any $\alpha>0$, there exists $C_{\alpha}$ such that

$$
|g(s)| \leq C_{\alpha} e^{\alpha s^{2}},
$$

for any $s \geq 0$.

- (H3) Define $k(s):=\frac{g(s)}{\sqrt{1+2 l^{\prime}\left(F^{2}(s)\right) F^{2}(s)}}$, then, for $N \geq 2$, there exists $s_{0}$ such that $\int_{0}^{s_{0}} k(r) d r=0$ and $k\left(s_{0}\right)>0$, while for $N=1$, there exists $s_{0}$ such that $\int_{0}^{s} k(r) d r<0$ for all $s \in\left(0, s_{0}\right), \int_{0}^{s_{0}} k(r) d r=0$ and $k\left(s_{0}\right)>0$;

- (H4) the function $l$ has the following regularity: $l \in C^{2}\left(\mathbf{R}^{+}, \mathbf{R}\right)$.

Then, Eq. (3) admits a solution $Q(x) \in H^{1}\left(\mathbf{R}^{N}\right)$ satisfying the following properties:

- $Q(x)>0$ for any $x \in \mathbf{R}^{N}$;

- $Q(x)$ is spherically symmetric;

- $Q(x)$ is smooth;

- $Q(x)$ and its derivatives up to order two are exponentially decaying.

Remark 1.2. An important thing to notice here is that the critical exponent $p^{*}$ varies accordingly to the growth of the function $l$. For example, in the case $l(v)=v$, the critical exponent is $p^{*}=3+\frac{4}{N-2}$ (see for example [6]).

Remark 1.3. We note that this type of result is very similar to the results that have been proven by Adachi and Watanabe [1]. We refer to [1] and the references therein for more details.

Another application of this dual approach involves the $p$-Laplacian. An example of results that we can prove with transformation theory is the following.

Theorem 1.4. Consider the following equation

$$
\Delta_{p} u+u \Delta_{p}|u|^{2}+|x|^{-\beta} g(u)=0, \quad \text { in } \Omega
$$

where $\beta<p, g: \mathbf{R}_{0}^{+} \rightarrow \mathbf{R}$ is continuous and $\Omega=\mathbf{R}^{n}$ if $\beta \leq 0$, while $\Omega=\mathbf{R}^{N} \backslash\{0\}$ if $\beta \in(0, p)$. Consider $T$, the solution of

$$
\left\{\begin{array}{l}
T^{\prime}(s)=\frac{1}{\left(1+2^{p-1} T^{p}(s)\right)^{1 / p}} \\
T(0)=0 .
\end{array}\right.
$$

Assume the following conditions on the nonlinear term $g$ :

- $(\mathrm{F} 0) f(u):=\frac{g(T(u))}{\left(1+2^{p-1} T(u)^{p}\right)^{1 / p}}$ is continuous in $\mathbf{R}_{0}^{+}$;

- (F1) there exists $a>0$ and $q>1$ such that $\lim _{u \rightarrow 0^{+}} u^{1-q} f(u)=-a$;

- (F2) there exists $u^{*}>0$ such that $f\left(u^{*}\right) \geq 0$ and $\int_{0}^{u^{*}} f(t) d t>0$;

- (F3) $\lim _{u \rightarrow \infty} u^{1-p_{\beta}^{*}} f(u)=0, \quad d_{\beta}^{*}:=p \frac{n-\beta}{n-p}$.

Then, Eq. (4), with $\beta<p$, admits a radial ground state $u \in \mathcal{D}_{\text {rad }}^{1, p}\left(\mathbf{R}^{N}\right) \cap L^{q}\left(\mathbf{R}^{n}\right)$ bounded by $u^{*}$. Moreover,

- $u \in C_{l o c}^{1, \theta}\left(\mathbf{R}^{N} \backslash\{0\}\right)$ for some $\theta \in(0,1)$;

- $|D u|^{p-2} D u \in C^{1}\left(\mathbf{R}^{N} \backslash\{0\}\right)$ and u solves (4) pointwise in $\mathbf{R}^{N} \backslash\{0\}$;

- $|D u(x)| \rightarrow 0$ as $|x| \rightarrow+\infty$ and $\left|D\left(T^{-1} u\right)(x)\right|=O\left(|x|^{-(N-1) /(p-1)}\right)$ as $|x| \rightarrow 0$; 
- $u$ is continuous at $x=0,(x, D u(x))_{\mathbf{R}^{N}} \leq 0$ in $\mathbf{R}^{N} \backslash\{0\}$, and $\|u\|_{\infty}=$ $u(0) \in\left(u_{0}, u^{*}\right]$, where $u_{0}:=\inf \left\{v>0: \int_{0}^{v} f(t) d t>0\right\}$;

- if $1<p \leq 2$, then $T^{-1}(u) \in H_{l o c}^{2, p}\left(\mathbf{R}^{N} \backslash\{0\}\right)$; if, furthermore, $\beta<\frac{N}{p^{\prime}}$, then $T^{-1}(u) \in H_{l o c}^{2, p}\left(\mathbf{R}^{N}\right)$.

If $1<q<p$, then $u$ s compactly supported in $\mathbf{R}^{N}$ and is a fast decay solution of (4) such that $T^{-1}(u) \in H^{1, p}\left(\mathbf{R}^{N}\right)$. Furthermore, $u$ has the regularity in $\mathbf{R}^{N}$ as described in the following table

\begin{tabular}{lll}
\hline & $1<p \leq 2$ & $p>2$ \\
\hline$\beta<1$ & $C^{2}\left(\mathbf{R}^{N}\right)$ & $C^{1}\left(\mathbf{R}^{N}\right)$ \\
$1 \leq \beta<p$ & $C_{l o c}^{0,(p-\beta) /(p-1)}\left(\mathbf{R}^{N}\right)$ & $C_{l o c}^{0,(p-\beta) /(p-1)}\left(\mathbf{R}^{N}\right)$
\end{tabular}

While, if $q \geq p$, then $u$ is positive in $\mathbf{R}^{N}, u \in C^{2}\left(\mathbf{R}^{N} \backslash\{0\}\right)$ and $u$ has the following regularity in $\mathbf{R}^{N}$ as described in the following table

\begin{tabular}{lll}
\hline & $1<p \leq 2$ & $p>2$ \\
\hline$\beta<2-p$ & $C^{2}\left(\mathbf{R}^{N}\right)$ & $C^{2}\left(\mathbf{R}^{N}\right)$ \\
$\beta=2-p$ & $C^{2}\left(\mathbf{R}^{N}\right)$ & $C_{l o c}^{1,1}\left(\mathbf{R}^{N}\right)$ \\
$2-p<\beta<1$ & $C^{2}\left(\mathbf{R}^{N}\right)$ & $C_{l o c}^{1,(1-\beta) /(p-1)}\left(\mathbf{R}^{N}\right)$ \\
$1 \leq \beta<p$ & $C_{l o c}^{0,(p-\beta) /(p-1)}\left(\mathbf{R}^{N}\right)$ & $C_{l o c}^{0,(p-\beta) /(p-1)}\left(\mathbf{R}^{N}\right)$
\end{tabular}

Moreover, $u$ is a fast decay solution of (4) and, in particular, $|x|^{(n-p) /(p-1)}$ $T^{-1}(u)$ is decreasing in $[R, \infty)$ for $R$ sufficiently large and approaches a limit $l \geq 0$ as $r \rightarrow+\infty$. While $l>0$, then $T^{-1}(u) \in H^{1, p}\left(\mathbf{R}^{N}\right)$ if and only if $N>p^{2}$; while, if $l=0$ and $N>p^{2}$, then $T^{-1}(u) \in H^{1, p}\left(\mathbf{R}^{N}\right)$.

Remark 1.5. The novelty of this result lies in the contemporary presence of both the terms $\Delta_{p} u$ and $u \Delta_{p} u^{2}$. In the case in which $u \Delta u^{2}$ is absent, we reduce our problem to Theorem 2.5 below proven by [16]. Instead, if the $\Delta_{p} u$ is absent, we can call $u^{2}=: v$ and apply again Theorem 2.5. When both the terms $\Delta_{p} u$ and $u \Delta_{p} u^{2}$ are present, Theorem 2.5 cannot be applied and one needs our approach.

Remark 1.6. We note that the transformation trick does not work if we aim to transform the $p$-Laplacian into the ordinary Laplacian. In fact, in that case, the transformation reduces to the identity and the PDE remains unchanged. This transformation trick seems therefore useful every time we want to remove a lower order coefficient in front of the main derivative term, but not when we want to modify the derivative term.

Since the dual approach seemed pretty successful in the stationary case, we may wonder what happens in the case of solutions with non trivial dynamics. We start by considering a quasilinear reaction-diffusion equation.

Theorem 1.7. Consider the following quasilinear heat equation

$$
u_{t}=\Delta u+u \Delta|u|^{2}+g(u)
$$


with initial condition $u(0)=u_{0} \geq 0$ and with $g(u) \leq 0$ for any $u \geq 0$ and 0 otherwise. We assume that $x \in M:=T^{N}$, the $N$-dimensional torus. If $u_{0}=F\left(v_{0}\right)$, with $F$ such that

$$
\left\{\begin{array}{l}
F^{\prime}(s)=\frac{1}{\sqrt{1+2 F^{2}(s)}} \\
F(0)=0 .
\end{array}\right.
$$

and with $v_{0}=F^{-1}\left(u_{0}\right) \in H^{s}(M), s>\frac{N}{2}+1$, then, there exists a unique solution such that

$$
v=F^{-1}(u) \in C\left([0, T), H^{s}(M)\right) \cap C^{\infty}((0, T) \times M),
$$

which persists as long as $\|u(t)\|_{C^{r}}$ is bounded, given $r>0$.

Remark 1.8. This result is a simple application of well known theorems, after the change of variables performed through $F$. However, we decided to discuss this theorem, because important to show the wide applicability of the dual approach. We refer to [21] for more possible applications of this method in the setting of quasilinear reaction-diffusion systems.

More interesting is to understand what happens to the complete evolution of the quasilinear Schrödinger equation.

Theorem 1.9. Suppose that $u$ solves the equation

$$
i u_{t}=-\Delta u-u \Delta|u|^{2}-g\left(|u|^{2}\right) u
$$

with hypotheses on the nonlinearity $g$ which ensure local well-posedness. Suppose that there exists a holomorphic transformation $F$ that eliminates the gradient terms in this equation. Then, $u$ is real up to a phase shift constant in space and time. Moreover, $u$ is stationary.

Remark 1.10. Essentially, this means that the method fails in the nonstationary case for equation of evolution type, which mix real and imaginary parts.

One might still wonder if the reason is that the requirement of holomorphicity for $F$ in the previous theorem is too strict. The answer is in the following theorem.

Theorem 1.11. Suppose that $u$ solves the equation

$$
i u_{t}=-\Delta u-u \Delta|u|^{2}-g\left(|u|^{2}\right) u
$$

with hypotheses on the nonlinearity $g$ which ensure local well-posedness. Suppose that there exists a transformation $F$, not necessarily holomorphic, that eliminates the gradient terms in this equation. Then, $u$ is real up to a phase shift constant in space and time. Moreover, $u$ is stationary.

Remark 1.12. Note that the only difference between these two theorems is the regularity requirement on the transformation.

This implies that in general, if one studies the complete evolution, it is not possible, through this strategy, to simplify the original quasilinear problem to a semilinear one. 
Remark 1.13. We think that the dual approach can be potentially adapted to more general combinations of derivative terms and nonlinear terms. For example, it would be interesting to understand what happens for non-local equations and for more general evolution problems.

The remaining part of the paper is organized as follows. In Sect. 2, we collect some results concerning the inverse of partitioned matrices, the CauchyRiemann equations, the results of Berestycki and Lions [2] and Berestycki et al. [3] on the semilinear problem, a result of the survey of Pucci and Servadei [16] on the $p$-Laplacian and a result concerning strong parabolic equations present in [21]. In Sect. 3, we prove Theorem 1.1. In Sect. 4, we prove Theorem 1.4. In Sect. 5, we prove Theorem 1.7. In Sect. 6, we prove Theorem 1.9. In Sect. 7, we prove Theorem 1.11.

\section{Preliminaries}

We collect some results which we will need later in our discussion and which help us to frame our problem in the literature.

\subsection{The inverse of a partitioned matrix}

In the proof of Theorem 1.11, we need an expression for the inverse of a partitioned matrix. In particular, we need the following lemma.

Lemma 2.1. Consider an $N \times N$ matrix $B$ partitioned in the following way:

$$
B:=\left(\begin{array}{ll}
B_{11} & B_{12} \\
B_{21} & B_{22}
\end{array}\right) .
$$

Then, if the inverse exists, it takes the following form:

$$
B^{-1}:=\left(\begin{array}{ll}
\left(B_{11}-B_{12} B_{22}^{-1} B_{21}\right)^{-1} & \left(-B_{11}^{-1} B_{12}\left(B_{22}-B_{21} B_{11}^{-1} B_{12}\right)^{-1}\right. \\
\left(-B_{22}^{-1} B_{21}\left(B_{11}-B_{12} B_{22}^{-1} B_{21}\right)^{-1}\right. & \left(B_{22}-B_{21} B_{11}^{-1} B_{12}\right)^{-1}
\end{array}\right) .
$$

\subsection{The Cauchy-Riemann equations}

The Cauchy-Riemann equations are a necessary condition that a complex function must satisfy to be differentiable. Here is the precise statement:

Theorem 2.2. Suppose that $f^{\prime}\left(z_{0}\right)$ exists at a point $z_{0} \in \mathcal{O} \subset \mathbb{C}$. Then the following equation must be satisfied:

$$
\frac{\partial}{\partial \bar{z}} f=0
$$

This condition is called Cauchy-Riemann equations.

Proof. The proof is a simple calculation. We refer to [17] for a proof of this well known fact.

Remark 2.3. The Cauchy-Riemann equations can be rewritten in several different forms, which all can be deducted by the previous condition. We refer to [17] for more details. 
At least at the level of continuity, we can identify $\mathbb{C}$ and $\mathbb{R}^{2}$, through the following transformation

$$
u \mapsto^{i}\left(u_{1}, u_{2}\right)^{T} \mapsto^{F}\left(v_{1}, v_{2}\right)^{T} \mapsto^{i^{-1}} v .
$$

We remind that if $F=\left(F_{1}, F_{2}\right)$ and $F_{1}, F_{2}$ are real differentiable, then $i^{-1} \circ F$ is real differentiable, but not necessarily complex differentiable. In fact $\left(i^{-1} \circ\right.$ $F \circ i) u$ is complex differentiable if and only if Cauchy-Riemann equations are satisfied.

\subsection{The semilinear problem}

The following is a classical result due to Berestycki and Lions [2] and Berestycki et al. [3].

Theorem 2.4. Consider the following equation:

$$
-\Delta v=k(v), \quad v \in H,
$$

which is the Euler-Lagrange equation of

$$
J(v):=\frac{1}{2} \int_{\mathbf{R}^{n}} d x|\nabla v|^{2}-\int_{\mathbf{R}^{n}} d x K(v),
$$

where $K(t)=\int_{0}^{t} k(s) d s$ is differentiable. Assume the following conditions:

- (A) $k(s) \in C\left(\mathbf{R}^{+}, \mathbf{R}\right)$ and $k(s)=0$ for $s \in \mathbf{R}^{-}$;

- (B) for $N \geq 3$

$$
-\infty<\liminf _{s \rightarrow 0} \frac{k(s)}{s} \leq \limsup _{s \rightarrow 0} \frac{k(s)}{s}=-\nu<0,
$$

while for $N=1,2$,

$$
\lim _{s \rightarrow 0} \frac{k(s)}{s}=-\nu<0
$$

- (C) for $N \geq 3, \lim _{s \rightarrow+\infty} \frac{k(s)}{s^{N+2}}=0$, while for $N=2$, for any $\alpha>0$, there exists $C_{\alpha}$ such that

$$
|k(s)| \leq C_{\alpha} e^{\alpha s^{2}}
$$

for any $s \geq 0$;

- (D) for $N \geq 2$, there exists $s_{0}$ such that $K\left(s_{0}\right)=0$ and $k\left(s_{0}\right)>0$, while for $N=1$, there exists $s_{0}$ such that $K(s)<0$ for all $s \in\left(0, s_{0}\right)$, $K\left(s_{0}\right)=0$ and $k\left(s_{0}\right)>0$.

Define

$$
m:=\inf \{J(v) \mid v \in H \backslash\{0\},-\Delta v=k(v)\} .
$$

Then $m>0$ and there exists a least energy solution $Q(x)$ of Eq. (6) such that

- $Q(x)>0$ for any $x \in \mathbf{R}^{N}$;

- $Q(x)$ is spherically symmetric;

- $Q(x)$ is smooth;

- $Q(x)$ and its derivatives up to order two are exponentially decaying. 


\subsection{The $p$-Laplacian with weights}

There is a wide literature concerning the $p$-Laplace operator. Here, we report a result exposed in the survey [16]. We refer to [16] for a more detailed literature review and for different results.

Theorem 2.5. Consider the following equation

$$
\Delta_{p} u+|x|^{-\beta} f(u)=0, \quad \text { in } \Omega
$$

where $\beta<p, f: \mathbf{R}_{0}^{+} \rightarrow \mathbf{R}$ is continuous and $\Omega=\mathbf{R}^{n}$ if $\beta \leq 0$, while $\Omega=$ $\mathbf{R}^{N} \backslash\{0\}$ if $\beta \in(0, p)$. Assume the following conditions on the nonlinear term $f:$

- (F0) $f$ is continuous in $\mathbf{R}_{0}^{+}$;

- (F1) there exists $a>0$ and $q>1$ such that $\lim _{u \rightarrow 0^{+}} u^{1-q} f(u)=-a$;

- (F2) there exists $u^{*}>0$ such that $f\left(u^{*}\right) \geq 0$ and $\int_{0}^{u^{*}} f(t) d t>0$;

- (F3) $\lim _{u \rightarrow \infty} u^{1-p_{\beta}^{*}} f(u)=0, \quad d_{\beta}^{*}:=p \frac{n-\beta}{n-p}$.

Then, Eq. (8), with $\beta<p$, admits a radial ground state $u \in \mathcal{D}_{\text {rad }}^{1, p}\left(\mathbf{R}^{N}\right) \cap$ $L^{q}\left(\mathbf{R}^{N}\right)$ bounded by $u^{*}$. Moreover,

- $u \in C_{l o c}^{1, \theta}\left(\mathbf{R}^{N} \backslash\{0\}\right)$ for some $\theta \in(0,1)$;

- $|D u|^{p-2} D u \in C^{1}\left(\mathbf{R}^{N} \backslash\{0\}\right)$ and u solves (8) pointwise in $\mathbf{R}^{N} \backslash\{0\}$;

- $|D u(x)| \rightarrow 0$ as $|x| \rightarrow+\infty$ and $|D u(x)|=O\left(|x|^{-(N-1) /(p-1)}\right)$ as $|x| \rightarrow 0$;

- $u$ is continuous at $x=0,(x, D u(x))_{\mathbf{R}^{N}} \leq 0$ in $\mathbf{R}^{N} \backslash\{0\}$, and $\|u\|_{\infty}=$ $u(0) \in\left(u_{0}, u^{*}\right]$, where $u_{0}:=\inf \left\{v>0: \int_{0}^{v} f(t) d t>0\right\}$;

- if $1<p \leq 2$, then $u \in H_{\text {loc }}^{2, p}\left(\mathbf{R}^{N} \backslash\{0\}\right)$; if, furthermore, $\beta<\frac{n}{p^{\prime}}$, then $u \in H_{l o c}^{2, p}\left(\mathbf{R}^{N}\right)$.

If $1<q<p$, then $u$ s compactly supported in $\mathbf{R}^{N}$ and it is a fast decay solution of (8) of class $H^{1, p}\left(\mathbf{R}^{N}\right)$. Furthermore, $u$ has the regularity in $\mathbf{R}^{N}$ as described in the following table

\begin{tabular}{lll}
\hline & $1<p \leq 2$ & $p>2$ \\
\hline$\beta<1$ & $C^{2}\left(\mathbf{R}^{N}\right)$ & $C^{1}\left(\mathbf{R}^{N}\right)$ \\
$1 \leq \beta<p$ & $C_{l o c}^{0,(p-\beta) /(p-1)}\left(\mathbf{R}^{N}\right)$ & $C_{l o c}^{0,(p-\beta) /(p-1)}\left(\mathbf{R}^{N}\right)$
\end{tabular}

While, if $q \geq p$, then $u$ is positive in $\mathbf{R}^{N}, u \in C^{2}\left(\mathbf{R}^{N} \backslash\{0\}\right)$ and $u$ has the following regularity in $\mathbf{R}^{N}$ as described in the following table

\begin{tabular}{lll}
\hline & $1<p \leq 2$ & $p>2$ \\
\hline$\beta<2-p$ & $C^{2}\left(\mathbf{R}^{N}\right)$ & $C^{2}\left(\mathbf{R}^{N}\right)$ \\
$\beta=2-p$ & $C^{2}\left(\mathbf{R}^{N}\right)$ & $C_{l o c}^{1,1}\left(\mathbf{R}^{N}\right)$ \\
$2-p<\beta<1$ & $C^{2}\left(\mathbf{R}^{N}\right)$ & $C_{l o c}^{1,(1-\beta) /(p-1)}\left(\mathbf{R}^{N}\right)$ \\
$1 \leq \beta<p$ & $C_{l o c}^{0,(p-\beta) /(p-1)}\left(\mathbf{R}^{N}\right)$ & $C_{l o c}^{0,(p-\beta) /(p-1)}\left(\mathbf{R}^{N}\right)$
\end{tabular}

Moreover, $u$ is a fast decay solution of (8) and, in particular, $|x|^{(n-p) /(p-1)}$ $u$ is decreasing in $[R, \infty)$ for $R$ sufficiently large and approaches a limit $l \geq 0$ as $r \rightarrow+\infty$. While $l>0$, then $u \in H^{1, p}\left(\mathbf{R}^{N}\right)$ if and only if $N>p^{2}$; while, if $l=0$ and $N>p^{2}$, then $u \in H^{1, p}\left(\mathbf{R}^{N}\right)$. 


\subsection{Strong parabolic quasilinear equations}

In this subsection, we collect some results about strong parabolic equations. We start with the definition of strong parabolicity.

Definition 2.6. Consider the following system

$$
\frac{\partial u}{\partial t}=\sum_{j, k=1}^{N} A^{j k}\left(t, x, D_{x}^{1} u\right) \partial_{j} \partial_{k} u+B\left(t, x, D_{x}^{1} u\right) .
$$

We say that this system is strongly parabolic if it satisfies the following condition

$$
\sum_{j, k=1}^{N} A^{j k}\left(t, x, D_{x}^{1}(u)\right) \xi_{j} \xi_{k} \geq C_{0}|\xi|^{2} I d_{K \times K},
$$

where we say that a pair of symmetric $K \times K$ matrices $A_{1}$ and $A_{2}$ satisfy $A_{1} \geq A_{2}$, if and only if $A_{1}-A_{2}$ is a positive semidefinite matrix.

Concerning strong parabolic equations, we have the following theorem from [21].

Proposition 2.7. Assume that the system

$$
\frac{\partial u}{\partial t}=\sum_{j, k=1}^{N} A^{j k}(t, x, u) \partial_{j} \partial_{k} u+B(t, x, u)
$$

is strongly parabolic and that $u(0)=u_{0}$. Here, $u$ takes values in $\mathbf{R}^{K}$ and $A^{j k}$ is a symmetric $K \times K$ matrix. We assume that $A^{j k}$ and $B$ are smooth in their arguments. We assume that $x \in M=T^{N}$, the $N$-dimensional torus. If $u_{0} \in H^{s}(M), s>\frac{N}{2}+1$, then there exists a unique solution such that

$$
u \in C\left([0, T), H^{s}(M)\right) \cap C^{\infty}((0, T) \times M),
$$

which persists as long as $\|u(t)\|_{C^{r}}$ is bounded, given $r>0$.

\section{Proof of Theorem 1.1}

In this section, we prove Theorem 1.1. Therefore, consider the equation

$$
\Delta u-u l^{\prime}\left(|u|^{2}\right) \Delta l\left(|u|^{2}\right)+\omega u+f\left(|u|^{2}\right) u=0 .
$$

Use the transformation $u=F(v)$ with $F: \mathbf{R}^{+} \rightarrow \mathbf{R}$. Plugging this inside each term, we get the following. First:

$$
\frac{\partial}{\partial x_{j}} l\left(|u|^{2}\right)=2 F(v) \frac{\partial}{\partial x_{j}} F(v) l^{\prime}\left(|u|^{2}\right)=2 F(v) F^{\prime}(v) l^{\prime}\left(|u|^{2}\right) \frac{\partial}{\partial x_{j}} v .
$$

Taking another derivative, we get

$$
\begin{aligned}
\frac{\partial}{\partial x_{j}} \frac{\partial}{\partial x_{j}} l\left(|u|^{2}\right) & =\frac{\partial}{\partial x_{j}}\left(2 F(v) F^{\prime}(v) l^{\prime}\left(|u|^{2}\right) \frac{\partial}{\partial x_{j}} v\right) \\
& =2\left(F^{\prime}\right)^{2} l^{\prime}\left(F^{2}\right) \frac{\partial}{\partial x_{j}} v \frac{\partial}{\partial x_{j}} v+\left(2 F F^{\prime}\right)^{2} l^{\prime \prime}\left(F^{2}\right) \frac{\partial}{\partial x_{j}} v \frac{\partial}{\partial x_{j}} v
\end{aligned}
$$




$$
+2 F l^{\prime}\left(F^{2}\right) F^{\prime \prime} \frac{\partial}{\partial x_{j}} v \frac{\partial}{\partial x_{j}} v+2 F F^{\prime} l^{\prime}\left(F^{2}\right) \frac{\partial^{2}}{\partial x_{j}^{2}} v
$$

Therefore

$$
\begin{aligned}
& \Delta l\left(|u|^{2}\right)=|\nabla v|^{2}\left(2\left(F^{\prime}\right)^{2} l^{\prime}\left(F^{2}\right)+\left(2 F F^{\prime}\right)^{2} l^{\prime \prime}\left(F^{2}\right)\right. \\
& \left.+2 F l^{\prime}\left(F^{2}\right) F^{\prime \prime}\right)+2 F F^{\prime} l^{\prime}\left(F^{2}\right) \Delta v .
\end{aligned}
$$

Plugging this inside Eq. (9), we get

$$
\begin{aligned}
& -\left(F^{\prime \prime}|\nabla v|^{2}+F^{\prime} \Delta v\right)-F l^{\prime}\left(F^{2}\right)\left(2\left(F^{\prime}\right)^{2} l^{\prime}\left(F^{2}\right)\right. \\
& \left.\quad+\left(2 F F^{\prime}\right)^{2} l^{\prime \prime}\left(F^{2}\right)+2 F l^{\prime}\left(F^{2}\right) F^{\prime \prime}\right)|\nabla v|^{2}+ \\
& 2 F l^{\prime}\left(F^{2}\right) F^{\prime \prime}+2 F F^{\prime} l^{\prime}\left(F^{2}\right) \Delta v+\omega F+f\left(|F(v)|^{2}\right) F(v)=0,
\end{aligned}
$$

which properly reorganized becomes:

$$
\begin{aligned}
& -\Delta v\left(F^{\prime}+2 F F^{\prime} l^{\prime}\left(F^{2}\right)\right)+\omega F+f\left(|F(v)|^{2}\right) F(v) \\
& -|\nabla v|^{2}\left(F^{\prime \prime}+2\left(F^{\prime}\right)^{2} l^{\prime}\left(F^{2}\right)+\left(2 F F^{\prime}\right)^{2} l^{\prime \prime}\left(F^{2}\right)+2 F l^{\prime}\left(F^{2}\right) F^{\prime \prime}\right)=0 .
\end{aligned}
$$

Our goal is to eliminate the second line of the previous equation. To do that, we need to find $F$ such that:

$$
F^{\prime \prime}\left(1+2 F^{2}\left(l^{\prime}\right)^{2}\left(F^{2}\right)\right)+2\left(l^{\prime 2}\left(F^{2}\right)\right) F\left(F^{\prime}\right)^{2}+4 F^{3} l^{\prime \prime}\left(F^{2}\right) l^{\prime}\left(F^{2}\right)\left(F^{\prime}\right)^{2} .
$$

We can rewrite this equation as

$$
-\frac{F^{\prime \prime}}{F^{\prime}}=\frac{2\left(l^{\prime}\right)^{2} F F^{\prime}+4 F^{3} l^{\prime \prime} l^{\prime}\left(F^{\prime}\right)^{2}}{1+2 F^{2}\left(l^{\prime 2}\right)},
$$

which can be rewritten as

$$
-\frac{F^{\prime \prime}}{F^{\prime}}=\frac{1}{2} \frac{\frac{d}{d v}\left(1+2 F^{2}\left(l^{\prime 2}\right)\right)}{1+2 F^{2}\left(l^{\prime 2}\right)} .
$$

Integrating this side by side, we get

$$
-\ln \left|F^{\prime}\right|=\frac{1}{2} \ln \left(1+2 F^{2}\left(l^{\prime}\right)^{2}\right)
$$

using the condition $F^{\prime}(0)=1$. Therefore, we have

$$
\left\{\begin{array}{l}
F^{\prime}=\frac{1}{\sqrt{1+2 F^{2}\left(l^{\prime}\right)^{2}}} \\
F(0)=0 .
\end{array}\right.
$$

If we can get a $F$ with good properties (see Lemma 3.1 below), we reduced Eq. (9) to the following semilinear equation

$$
-\left(\frac{1}{\sqrt{1+2 F^{2}\left(l^{\prime}\right)^{2}}}+\frac{2 F^{2}\left(l^{\prime}\right)^{2}}{\sqrt{1+2 F^{2}\left(l^{\prime}\right)^{2}}}\right) \Delta v=f\left(F^{2}(v)\right) F(v)-\omega F(v),
$$

which can be simplified to

$$
-\Delta v=\frac{f\left(F^{2}(v)\right) F(v)-\omega F(v)}{\sqrt{1+2 F^{2}\left(l^{\prime}\right)^{2}}} .
$$

Now, we have to analyze the solutions of the ODE (10). 
Lemma 3.1. The solution $F$ of the equation

$$
\left\{\begin{array}{l}
F^{\prime}=\frac{1}{\sqrt{1+2\left(l^{\prime}\right)^{2} F^{2}}} \\
F(0)=0 .
\end{array}\right.
$$

satisfies the following properties:

- F is uniquely defined, smooth and invertible;

- $\left|F^{\prime}(v)\right| \leq 1$, for all $v \in \mathbf{R}$;

- $\frac{F(v)}{v} \rightarrow 1$ as $v \rightarrow 0$.

Proof. The proof is straightforward and corresponding to Lemma 2.1 of [5].

As an application of Lemma 3.1, we have that

$$
k(v):=\frac{f\left(F^{2}(v)\right) F(v)-\omega F(v)}{\sqrt{1+2 F^{2}\left(l^{\prime}\right)^{2}}}
$$

satisfies the hypotheses of Theorem 2.4. Therefore, by Theorem 2.4, we obtain the conclusion of Theorem 1.1.

Remark 3.2. See also [1] for completeness.

\section{Proof of Theorem 1.4}

In this section, we prove Theorem 1.4. Therefore, consider the equation

$$
\Delta_{p} u+u \Delta_{p} u^{2}+|x|^{-\beta} g(u)=0 .
$$

Again, we try to find a transformation of the form $u=T(v)$ with $T: \mathbf{R}^{+} \rightarrow \mathbf{R}$ which exploits the semilinear structure of our problem. We get

$$
\nabla u=\nabla(T(v))=T^{\prime} \nabla v
$$

We study the transformed equation term by term. First, we analyze the $p$ Laplacian term:

$$
\begin{aligned}
\Delta_{p} u= & \operatorname{div}\left(|\nabla u|^{p-2} \nabla u\right)=\operatorname{div}\left(\left|T^{\prime}\right|^{p-2} T^{\prime}|\nabla v|^{p-2} \nabla v\right) \\
= & \frac{\partial}{\partial x_{j}}\left(\left|T^{\prime}\right|^{p-2} T^{\prime}|\nabla v|^{p-2} \frac{\partial v}{\partial x_{j}}\right) \\
= & \left.T^{\prime}\right|^{p-2} T^{\prime}|\nabla v|^{p-2} \Delta v+\left|T^{\prime}\right|^{p-2} T^{\prime \prime}|\nabla v|^{p-2} \frac{\partial v}{\partial x_{j}} \frac{\partial v}{\partial x_{j}} \\
& \quad+(p-2)\left|T^{\prime}\right|^{p-3} T^{\prime \prime}|\nabla v|^{p-2} T^{\prime} \frac{T^{\prime}}{\left|T^{\prime}\right|} \frac{\partial v}{\partial x_{j}} \frac{\partial v}{\partial x_{j}} \\
& \quad+\left|T^{\prime}\right|^{p-2} T^{\prime} \frac{\partial}{\partial x_{j}}\left(\left|\frac{\partial}{\partial x_{l}} v\right|^{p-2}\right)^{\frac{p-2}{2}} \frac{\partial v}{\partial x_{j}} \\
= & \left.T^{\prime}\right|^{p-2} T^{\prime}|\nabla v|^{p-2} \Delta v+\left|T^{\prime}\right|^{p-2} T^{\prime \prime}|\nabla v|^{p-2}|\nabla v|^{2} \\
\quad & \quad+\left|T^{\prime}\right| p^{p-2} T^{\prime}|\nabla v|^{p-2}|\nabla v|^{2}+\left|T^{\prime}\right|^{p-2} T^{\prime} \nabla|\nabla v|^{p-2} \cdot \nabla v \\
= & \left.T^{\prime}\right|^{p-2} T^{\prime}|\nabla v|^{p-2} \Delta v+\left|T^{\prime}\right|^{p-2} T^{\prime \prime}|\nabla v|^{p}
\end{aligned}
$$




$$
\begin{array}{r}
\left.+\left|T^{\prime}\right|^{p-2} T^{\prime \prime}|\nabla v|^{p}+\left|T^{\prime}\right|^{p-2} T^{\prime} d i v|\nabla v|^{p-2} \cdot \nabla v\right)-\left|T^{\prime}\right|^{p-2} T^{\prime} \Delta v \\
=\left|T^{\prime}\right|^{p-2} T^{\prime \prime}|\nabla v|^{p}+\left|T^{\prime}\right|^{p-2} T^{\prime \prime}|\nabla v|^{p}+\left|T^{\prime}\right|^{p-2} T^{\prime} \operatorname{div}\left(|\nabla v|^{p-2} \cdot \nabla v\right)
\end{array}
$$

Therefore:

$$
\Delta_{p} u=\left|T^{\prime}\right|^{p-2} T^{\prime} \Delta_{p} v+(p-1)|\nabla v|^{p}\left|T^{\prime}\right|^{p-2} T^{\prime \prime} .
$$

Second, we consider $\Delta_{p} u^{2}$ and get

$$
\begin{gathered}
\Delta_{p} u^{2}=\operatorname{div}\left(\left|2 T T^{\prime} \nabla v\right|^{p-2} 2 T T^{\prime} \nabla v\right)=2^{p-1}\left|T T^{\prime}\right|^{p-2} T T^{\prime} \Delta_{p} v \\
+2^{p-1}(p-1)|\nabla v|^{p}\left|T T^{\prime}\right|^{p-2}\left(T T^{\prime}\right)^{\prime} .
\end{gathered}
$$

Putting these two computations together, we get

$$
\begin{aligned}
& \Delta_{p} u+u \Delta_{p} u^{2}+|x|^{-\beta} g(u)=\left|T^{\prime}\right|^{p-2} T^{\prime} \Delta_{p} v+(p-1)|\nabla v|^{p}\left|T^{\prime}\right|^{p-2} T^{\prime \prime} \\
&+T\left(2^{p-1}\left|T T^{\prime}\right|^{p-2} T T^{\prime} \Delta_{p} v+2^{p-1}(p-1)|\nabla v|^{p}\left|T T^{\prime}\right|^{p-2}\left(T T^{\prime}\right)^{\prime}\right)+|x|^{-\beta} g(u) \\
&= \Delta_{p} v\left(\left|T^{\prime}\right|^{p-2} T^{\prime}+2^{p-1}\left|T T^{\prime}\right|^{p-2} T T^{\prime}\right) \\
&+|\nabla v|^{p}\left((p-1)\left|T^{\prime}\right|^{p-2} T^{\prime \prime}+2^{p-1}(p-1)\left|T T^{\prime}\right|^{p-2}\left(T T^{\prime}\right)^{\prime}\right)+|x|^{-\beta} g(u) .
\end{aligned}
$$

Remark 4.1. Note that we have used that $T T^{\prime}$ is positive.

Now, we choose the following condition for the transformation $T$ :

$$
\left|T^{\prime}\right|^{p-2} T^{\prime \prime}+2^{p-1}\left|T T^{\prime}\right|^{p-2}\left(T T^{\prime}\right)^{\prime}=0,
$$

in order to eliminate the coefficient of $|\nabla v|^{p}$. We get

$$
T^{\prime \prime}\left(1+2^{p-1}|T|^{p}\right)+2^{p-1}|T|^{p-2} T T^{\prime \prime}=0 .
$$

Dividing by $T^{\prime}$ and by $1+2^{p-1}|T|^{p}$, we obtain

$$
\frac{-T^{\prime \prime}}{T^{\prime}}=\frac{2^{p-1}|T|^{p-2} T}{1+2^{p-1}|T|^{p}}
$$

and so

$$
-p \frac{d}{d v} \log \left|T^{\prime}\right|=\frac{d}{d v} \log \left(1+2^{p-1}|T|^{p}\right) .
$$

The equation for $T$ becomes

$$
\left\{\begin{array}{l}
T^{\prime}(s)=\frac{1}{\left(1+2^{p-1} T^{p}(s)\right)^{1 / p}} \\
T(0)=0 .
\end{array}\right.
$$

With this condition, we get

$$
\Delta_{p} u+\Delta_{p} u^{2}+|x|^{-\beta} g(u)=\frac{1}{T^{\prime}} \Delta_{p} v+|x|^{-\beta} g(T(v))
$$

and so our original problem is reduced to

$$
\Delta_{p} v+|x|^{-\beta} \frac{g(T(v))}{\left(1+2^{p-1} T^{p}(v)\right)^{1 / p}}=0 .
$$


The solution $T$ has properties similar to the ones of the function $F$ described in Lemma 3.1 (see also [5]). Therefore, this equation falls in the hypotheses of Theorem 2.5 and so, by Theorem 2.5, we get the thesis. This concludes the proof of the theorem.

Remark 4.2. An interesting fact here is that, in absence of the extra term $u \Delta|u|^{2}$, the transformation $T$ reduces to the identity. In fact:

$$
\Delta_{p} u=\left|T^{\prime}\right|^{p-2} T^{\prime} \Delta_{p} v+(p-1)|\nabla v|^{p}\left|T^{\prime}\right|^{p-2} T^{\prime \prime}
$$

and so to get rid of the term with $|\nabla v|^{p}$, one needs $\left|T^{\prime}\right|=0$ or $T^{\prime \prime}=0$ and so with the use of the initial conditions one gets $T(u)=u$. Therefore, with this method, there is no way to reduce the $p$-Laplace equation to the 2-Laplace equation.

\section{Proof of Theorem 1.7}

In this section, we prove Theorem 1.7. Therefore, we consider the following quasilinear reaction-diffusion equation

$$
u_{t}=\Delta+u \Delta|u|^{2}+g(u) \text {. }
$$

Here, $g(u)$ satisfies the hypotheses of Theorem 1.7. We apply again the transformation $u=F(v)$ with $F: \mathbf{R}^{+} \rightarrow \mathbf{R}$. Similarly as in the previous sections, we get

$$
\begin{aligned}
u_{t} & =F^{\prime}(v) v_{t}, \\
\Delta u & =F^{\prime \prime}(v)|\nabla v|^{2}+F^{\prime} \Delta v .
\end{aligned}
$$

and

$$
\Delta|u|^{2}=|\nabla v|^{2}\left(2\left(F^{\prime}\right)^{2}+2 F F^{\prime \prime}\right)+2 F F^{\prime} \Delta v .
$$

Plugging this inside our equation, we get:

$F^{\prime}(v) v_{t}=F^{\prime \prime}(v)|\nabla v|^{2}+F^{\prime} \Delta v+F|\nabla v|^{2}\left(2\left(F^{\prime}\right)^{2}+2 F F^{\prime \prime}\right)+2 F F^{\prime} \Delta v+g(F(v))$.

Therefore, as before, if we take $F$ such that

$$
\left\{\begin{array}{l}
F^{\prime}(s)=\frac{1}{\sqrt{1+2\left(l^{\prime}\right)^{2} F^{2}(s)}} \\
F(0)=0
\end{array}\right.
$$

we reduce our equation to

$$
F^{\prime} v_{t}=F^{\prime}\left(1+2 F^{2}\right) \Delta v+g(F(v))
$$

and hence to

$$
v_{t}=\left(1+2 F^{2}\right) \Delta v+\sqrt{1+2\left(l^{\prime}\right)^{2} F^{2}} g(F(v)) .
$$

This equation satisfies the hypotheses of Proposition 2.7, with

$$
A^{j j}(t, x, v)=1+2 F^{2}(v)
$$

and

$$
A^{j k}(t, x, v)=0
$$


for $j \neq k, k=1, \ldots, N$ and $B(t, x, v):=\sqrt{1+2 F^{2}} g(F(v))$. Therefore, we get local existence in time for our problem.

Remark 5.1. To get global estimates in time at least in $L^{2}(M)$, we notice that, multiplying

$$
v_{t}=\left(1+2 F^{2}\right) \Delta v+\sqrt{1+2 F^{2}} g(F(v))
$$

by $v$ side by side, integrating by parts and using the periodicity of $M=\mathbf{T}^{N}$, we get

$$
\frac{1}{2} \partial_{t}\|v\|_{L^{2}(M)}^{2}=-\int_{M}\left(|\nabla v|^{2} \frac{\left(1+2 F^{2}\right)^{3 / 2}+4 v F}{\left(1+2 F^{2}\right)^{1 / 2}}+\sqrt{1+2 F^{2}} g(F(v))\right) .
$$

Now, if $g(F(v)) \leq 0$, we get

$$
\|v(t)\|_{L^{2}(M)}^{2} \leq\|v(0)\|_{L^{2}(M)}^{2},
$$

which gives a uniform in time global bound in $L^{2}(M)$.

\section{Proof of Theorem 1.9}

In this section, we prove Theorem 1.9. Therefore, we consider the equation:

$$
i u_{t}+\Delta u+u \Delta\left(|u|^{2}\right)+g\left(|u|^{2}\right) u=0 .
$$

Rewriting it in an expanded way, it becomes

$$
i u_{t}=-\Delta u-2 u|\nabla u|^{2}-2 u \Re(\bar{u} \Delta u)-g\left(|u|^{2}\right) u .
$$

Suppose that $F$ is a holomorphic complex function on all $\mathbf{C}$ and consider $u=F(v)$. Then we have

$$
i u_{t}=i F^{\prime}(v) v_{t}
$$

also

$$
\begin{aligned}
\Delta u & =\sum_{j} \partial_{x_{j}} \partial_{x_{j}}(F(v)) \\
& =\sum_{j} \frac{\partial}{\partial x_{j}}\left(F^{\prime}(v) \cdot \frac{\partial v}{\partial x_{j}}\right) \\
& =\sum_{j}\left(F^{\prime \prime}(v) \frac{\partial v}{\partial x_{j}}+F^{\prime} \frac{\partial^{2} v}{\partial x_{j}^{2}}\right) \\
& =F^{\prime \prime}(v)|\nabla v|^{2}+F^{\prime} \Delta v .
\end{aligned}
$$

Hence, the Eq. (15) for $u$ can be transformed to an equation for $v$, which is in fact

$$
\begin{aligned}
& -i F^{\prime}(v) v_{t}=-F^{\prime \prime}(v) \cdot|\nabla v|^{2}-F^{\prime}(v) \Delta v-2 F(v)\left|F^{\prime}(v)\right|^{2} . \\
& |\nabla v|^{2}-2 F(v) \Re\left(\bar{F} F^{\prime \prime}(v)|\nabla v|^{2}+\bar{F} F^{\prime} \Delta v\right),
\end{aligned}
$$

and so

$$
\begin{aligned}
-i F^{\prime}(v) v_{t}= & |\nabla v|^{2}\left[-F^{\prime \prime}(v)-2 F(v)\left|F^{\prime}(v)\right|^{2}-2 F \Re\left(\overline{F(v)} F^{\prime \prime}(v)\right)\right] \\
& -F^{\prime}(v) \Delta v-2 F(v) \Re\left(\overline{F(v)} F^{\prime}(v) \Delta v\right) .
\end{aligned}
$$

We want to eliminate the term with a gradient in the nonlinearity, therefore we need that the following complex ODE is satisfied:

$$
F^{\prime \prime}+2 F\left|F^{\prime}\right|^{2}+2 F \Re\left(\bar{F} F^{\prime \prime}\right)=0 .
$$


Now, we study the Eq. (18). Multiplying the equation by $\bar{F}$, then

$$
F^{\prime \prime} \bar{F}+2|F|^{2}\left|F^{\prime}\right|^{2}+2|F|^{2} \Re\left(\bar{F} F^{\prime \prime}\right)=0 .
$$

The second and third term are both real valued and this makes the first term real valued too, i.e., $F^{\prime \prime} \bar{F}$ is real. Let us exploit this condition. Since $F$ is holomorphic, then $\frac{\partial}{\partial \bar{z}} F=0$ and so $F=F(z)=a(z)+i b(z)$. The condition $\Im\left(F^{\prime \prime} \bar{F}\right)=0$ becomes

$$
0=\Im\left(F^{\prime \prime} \bar{F}\right)=b^{\prime \prime}(z) a(z)-a^{\prime \prime}(z) b(z) .
$$

We add and sum on both the sides the quantity $a^{\prime} b^{\prime}$ and get therefore

$$
\left(b^{\prime} a\right)^{\prime}=\left(a^{\prime} b\right)^{\prime}
$$

which, using the condition $0=F(0)=a(0)+i b(0)$, becomes

$$
b^{\prime} a=a^{\prime} b .
$$

Integrating by parts again, we get

$$
\ln |a|=\ln |b|+C
$$

with $C \in \mathbf{R}$. This implies that

$$
|a(z)|=\gamma|b(z)|
$$

for some $\gamma>0$ and so that $F(z)=b(z)(1 \pm i \gamma)$ or $F(z)=b(z) e^{i \theta}$ with

$$
\left\{\begin{array}{l}
b^{\prime}=\frac{1}{\sqrt{1+2 b^{2}}} \\
b(0)=0 .
\end{array}\right.
$$

and with $\theta \in[0,2 \pi)$. This implies that $u$ must be real up to a constant. Plugging this condition in Eq. (14), we get that $u_{t}=0$. Therefore $u$ must be stationary and real up to a phase shift. This completes the proof of the theorem.

Remark 6.1. This result is, in some sense, to be expected, because the request of being holomorphic to a complex function can be thought as a reduction of dimensionality of that complex function. This does not affect the elliptic problem, where the solution is essentially real and so lower dimensional, but does affect the complete evolution, which is fully complex.

\section{Proof of Theorem 1.11}

In this section, we give a complete proof of Theorem 1.11. Consider the equation

$$
i u_{t}=-\Delta u-u \Delta|u|^{2}+\lambda|u|^{p-1} u .
$$

We decompose the solution $u$ and the equation into real and imaginary part. Therefore, the system satisfied by $\left(u_{1}, u_{2}\right)$ with $u=u_{1}+i u_{2}$, is $\left\{\begin{array}{c}-u_{1 t}=-\Delta u_{1}-2 u_{1}\left(\left|\nabla u_{1}\right|^{2}+\left|\nabla u_{2}\right|^{2}\right)-2 u_{1}\left(u_{1} \Delta u_{1}+u_{2} \Delta u_{2}\right)+\lambda\left|u_{1}^{2}+u_{2}^{2}\right|^{p-1} u_{1} \\ u_{2 t}=-\Delta u_{2}-2 u_{2}\left(\left|\nabla u_{1}\right|^{2}+\left|\nabla u_{2}\right|^{2}\right)-2 u_{2}\left(u_{1} \Delta u_{1}+u_{2} \Delta u_{2}\right)+\lambda\left|u_{1}^{2}+u_{2}^{2}\right|^{p-1} u_{2}\end{array}\right.$ 
Now, we use the transformation:

$$
\left\{\begin{array}{l}
u_{1}=F(v, w) \\
u_{2}=G(v, w)
\end{array}\right.
$$

We concentrate on one term at the time. First, we compute the time derivative:

$$
u_{1 t}=F_{v} v_{t}+F_{w} w_{t}
$$

and similarly

$$
u_{2 t}=G_{v} v_{t}+G_{w} w_{t}
$$

Second, the Laplacian:

$$
\begin{aligned}
\Delta u_{1} & =\sum_{i=1}^{n} \frac{\partial}{\partial x_{i}}\left(\frac{\partial}{\partial x_{i}} F(v, w)\right) \\
& =\sum_{i=1}^{n} \frac{\partial}{\partial x_{i}}\left(\frac{\partial}{\partial x_{i}} F(v, w)\right)=\sum_{i=1}^{n} \frac{\partial}{\partial x_{i}}\left(F_{v} v_{x_{i}}+F_{w} w_{x_{i}}\right) \\
& =\sum_{i=1}^{n} \frac{\partial}{\partial x_{i}}\left(F_{v v}\left|v_{x_{i}}\right|^{2}+F_{v w} v_{x_{i}} w_{x_{i}}+F_{w v} w_{x_{i}} v_{x_{i}}+F_{w w}\left|w_{x_{i}}\right|^{2}\right) \\
& =F_{v v}|\nabla v|^{2}+2 F_{v w} \nabla v \nabla w+F_{w w}|\nabla w|^{2}+F_{v} \Delta v+F_{w} \Delta w .
\end{aligned}
$$

Similarly:

$$
\Delta u_{2}=G_{v v}|\nabla v|^{2}+2 G_{v w} \nabla v \nabla w+G_{w w}|\nabla w|^{2}+G_{v} \Delta v+G_{w} \Delta w .
$$

Then, the gradient terms:

$$
\left|\nabla u_{1}\right|^{2}=F_{v}^{2}|\nabla v|^{2}+2 F_{v} F_{w} \nabla v \nabla w+F_{w}^{2}|\nabla w|^{2}
$$

and also

$$
\left|\nabla u_{2}\right|^{2}=G_{v}^{2}|\nabla v|^{2}+2 G_{v} G_{w} \nabla v \nabla w+G_{w}^{2}|\nabla w|^{2}
$$

We can plug everything inside the equation and get the system:

$$
\left\{\begin{array}{l}
-F_{v} v_{t}-F_{w} w_{t}=-\left(1+2 F^{2}\right)\left(F_{v v}|\nabla v|^{2}\right. \\
\left.\quad+2 F_{v w} \nabla v \nabla w+F_{w w}|\nabla w|^{2}+F_{v} \Delta v+F_{w} \Delta w\right) \\
\quad-2 F\left(\left(F_{v}^{2}+G_{v}^{2}\right)|\nabla v|^{2}+2\left(F_{v} F_{w}+G_{v} G_{w}\right) \nabla v \nabla w+\left(F_{w}^{2}+G_{w}^{2}\right)|\nabla w|^{2}\right) \\
\quad-2 F G\left(G_{v v}|\nabla v|^{2}+2 G_{v w} \nabla v \nabla w+G_{w w}|\nabla w|^{2}\right. \\
\left.\quad+G_{v} \Delta v+G_{w} \Delta w\right)+\lambda\left|F^{2}+G^{2}\right|^{\frac{p-1}{2}} F \\
G_{v} v_{t}+G_{w} w_{t}=-\left(1+2 G^{2}\right)\left(G_{v v}|\nabla v|^{2}+2 G_{v w} \nabla v \nabla w\right. \\
\left.\quad+G_{w w}|\nabla w|^{2}+G_{v} \Delta v+G_{w} \Delta w\right) \\
\quad-2 G\left(\left(F_{v}^{2}+G_{v}^{2}\right)|\nabla v|^{2}+2\left(F_{v} F_{w}+G_{v} G_{w}\right) \nabla v \nabla w+\left(F_{w}^{2}+G_{w}^{2}\right)|\nabla w|^{2}\right) \\
\quad-2 F G\left(F_{v v}|\nabla v|^{2}+2 F_{v w} \nabla v \nabla w+F_{w w}|\nabla w|^{2}\right. \\
\left.\quad+F_{v} \Delta v+F_{w} \Delta w\right)+\lambda\left|F^{2}+G^{2}\right|^{\frac{p-1}{2}} G .
\end{array}\right.
$$


Therefore, to eliminate the gradient terms we need to solve the solving quasilinear system of coupled PDEs:

$$
\left\{\begin{array}{c}
-F_{v v}\left(1+2 F^{2}\right)-2 F\left(F_{v}^{2}+G_{v}^{2}\right)-2 F G G_{v v}=0, \\
-2 F_{v w}\left(1+2 F^{2}\right)-4 F\left(F_{v} F_{w}+G_{v} G_{w}\right)-4 F G G_{v w}=0, \\
-F_{w w}\left(1+2 F^{2}\right)-2 F\left(F_{w}^{2}+G_{w}^{2}\right)-2 F G G_{w w}=0, \\
-G_{v v}\left(1+2 G^{2}\right)-2 G\left(F_{v}^{2}+G_{v}^{2}\right)-2 F G F_{v v}=0, \\
-2 G_{v w}\left(1+2 G^{2}\right)-4 F\left(F_{v} F_{w}+G_{v} G_{w}\right)-4 F G F_{v w}=0, \\
-G_{w w}\left(1+2 G^{2}\right)-2 G\left(F_{w}^{2}+G_{w}^{2}\right)-2 F G F_{w w}=0 .
\end{array}\right.
$$

We can rewrite this system in the following matrix form

$$
\begin{aligned}
& {\left[\begin{array}{cccccc}
-\left(1+2 F^{2}\right) & 0 & 0 & -2 F G & 0 & 0 \\
0 & -2\left(1+2 F^{2}\right) & 0 & 0 & -4 F G & 0 \\
0 & 0 & -\left(1+2 F^{2}\right) & 0 & 0 & -2 F G \\
-2 F G & 0 & 0 & -\left(1+2 G^{2}\right) & 0 & 0 \\
0 & -4 F G & 0 & 0 & -2\left(1+2 G^{2}\right) & 0 \\
0 & 0 & -2 F G & 0 & 0 & -\left(1+2 G^{2}\right)
\end{array}\right]\left[\begin{array}{c}
F_{v v} \\
F_{v w} \\
F_{w w} \\
G_{v v} \\
G_{v w} \\
G_{w w}
\end{array}\right]} \\
& =\left[\begin{array}{c}
2 F\left(F_{v}^{2}+G_{v}^{2}\right) \\
4 F\left(F_{v} F_{w}+G_{v} G_{w}\right) \\
2 F\left(F_{w}^{2}+G_{w}^{2}\right) \\
2 G\left(F_{v}^{2}+G_{v}^{2}\right) \\
4 G\left(F_{v} F_{w}+G_{v} G_{w}\right) \\
2 G\left(F_{w}^{2}+G_{w}^{2}\right)
\end{array}\right]
\end{aligned}
$$

We can rewrite this in a more compact form. Define

$$
\begin{aligned}
B:= & {\left[\begin{array}{ll}
B_{11} & B_{12} \\
B_{21} & B_{22}
\end{array}\right] } \\
= & {\left[\begin{array}{cccccc}
-\left(1+2 F^{2}\right) & 0 & 0 & -2 F G & 0 & 0 \\
0 & -2\left(1+2 F^{2}\right) & 0 & 0 & -4 F G & 0 \\
0 & 0 & -\left(1+2 F^{2}\right) & 0 & 0 & -2 F G \\
-2 F G & 0 & 0 & -\left(1+2 G^{2}\right) & 0 & 0 \\
0 & -4 F G & 0 & 0 & -2\left(1+2 G^{2}\right) & 0 \\
0 & 0 & -2 F G & 0 & 0 & -\left(1+2 G^{2}\right)
\end{array}\right], } \\
& F^{\prime \prime}:=\left[\begin{array}{c}
F_{v v} \\
F_{v w} \\
F_{w w} \\
G_{v v} \\
G_{v w} \\
G_{w w}
\end{array}\right]
\end{aligned}
$$

and

Our system takes the form:

$$
h:=\left[\begin{array}{c}
2 F\left(F_{v}^{2}+G_{v}^{2}\right) \\
4 F\left(F_{v} F_{w}+G_{v} G_{w}\right) \\
2 F\left(F_{w}^{2}+G_{w}^{2}\right) \\
2 G\left(F_{v}^{2}+G_{v}^{2}\right) \\
4 G\left(F_{v} F_{w}+G_{v} G_{w}\right) \\
2 G\left(F_{w}^{2}+G_{w}^{2}\right)
\end{array}\right]
$$

$$
B F^{\prime \prime}=h .
$$


Here $B_{11}=-\left(-1+2 F^{2}\right) M, B_{12}=B_{21}=-2 F G M$ and $B_{21}=-\left(-1+2 G^{2}\right) M$, where

$$
M:=\left[\begin{array}{lll}
1 & 0 & 0 \\
0 & 2 & 0 \\
0 & 0 & 1
\end{array}\right]
$$

This particular form turns out to be very helpful and the solution of this system is not unfeasible as it could seem at first sight. This matrix $B$ is partitioned into four squared diagonal blocks. We use Lemma 2.1, to compute the inverse of the matrix $B$ :

$B^{-1}:=\left(\begin{array}{ll}\left(B_{11}-B_{12} B_{22}^{-1} B_{21}\right)^{-1} & \left(-B_{11}^{-1} B_{12}\left(B_{22}-B_{21} B_{11}^{-1} B_{12}\right)^{-1}\right. \\ \left(-B_{22}^{-1} B_{21}\left(B_{11}-B_{12} B_{22}^{-1} B_{21}\right)^{-1}\right. & \left(B_{22}-B_{21} B_{11}^{-1} B_{12}\right)^{-1}\end{array}\right)$.

We compute entry by entry:

$$
\begin{aligned}
& \left(B_{11}-B_{12} B_{22}^{-1} B_{21}\right)^{-1}=\left(-\left(1+2 F^{2}\right) M-\frac{4 F^{2} G^{2}}{1+2 G^{2}} M M^{-1} M\right) \\
& =-\left(\frac{1+2\left(F^{2}+G^{2}\right)}{1+2 G^{2}}\right)^{-1} M^{-1}=-\frac{1+2 G^{2}}{1+2\left(F^{2}+G^{2}\right)}\left[\begin{array}{ccc}
1 & 0 & 0 \\
0 & 0.5 & 0 \\
0 & 0 & 1
\end{array}\right] .
\end{aligned}
$$

Now,

$$
\begin{aligned}
( & -B_{11}^{-1} B_{12}\left(B_{22}-B_{21} B_{11}^{-1} B_{12}\right)^{-1} \\
& =\frac{1}{1+2 F^{2}} M^{-1}(-2 F G) M\left(-\left(1+2 G^{2}\right) M-\frac{4 F^{2} G^{2}}{1+2 F^{2}} M M^{-1} M\right) \\
& =-\frac{2 F G}{1+2 F^{2}} \frac{1+2 F^{2}}{1+2\left[F^{2}+G^{2}\right]} M^{-1} \\
& =\frac{2 F G}{1+2\left(F^{2}+G^{2}\right)} M^{-1}=\frac{2 F G}{1+2\left(F^{2}+G^{2}\right)}\left[\begin{array}{ccc}
1 & 0 & 0 \\
0 & 0.5 & 0 \\
0 & 0 & 1
\end{array}\right] .
\end{aligned}
$$

Therefore, also

$$
\begin{aligned}
& \left(-B_{22}^{-1} B_{21}\left(B_{11}-B_{12} B_{22}^{-1} B_{21}\right)^{-1}=\frac{2 F G}{1+2\left(F^{2}+G^{2}\right)} M^{-1}\right. \\
& \quad=\frac{2 F G}{1+2\left(F^{2}+G^{2}\right)}\left[\begin{array}{ccc}
1 & 0 & 0 \\
0 & 0.5 & 0 \\
0 & 0 & 1
\end{array}\right] .
\end{aligned}
$$

And finally

$$
\begin{aligned}
& \left(B_{22}-B_{21} B_{11}^{-1} B_{12}\right)^{-1}=\left(-\left(1+2 G^{2}\right) M-\frac{4 F^{2} G^{2}}{1+2 F^{2}} M M^{-1} M\right) \\
& =-\left(\frac{1+2\left(F^{2}+G^{2}\right)}{1+2 F^{2}}\right)^{-1} M^{-1}=-\frac{1+2 F^{2}}{1+2\left(F^{2}+G^{2}\right)}\left[\begin{array}{ccc}
1 & 0 & 0 \\
0 & 0.5 & 0 \\
0 & 0 & 1
\end{array}\right] .
\end{aligned}
$$

We can rewrite our system as follows

$$
F^{\prime \prime}=B^{-1} h,
$$


with $B^{-1}$ taking the following form:

$$
B^{-1}:=\left[\begin{array}{c}
-\frac{1+2 G^{2}}{1+2\left(F^{2}+G^{2}\right)} M^{-1}-\frac{2 F G}{1+2\left(F^{2}+G^{2}\right)} M^{-1} \\
-\frac{2 F G}{1+2\left(F^{2}+G^{2}\right)} M^{-1}-\frac{1+2 F^{2}}{1+2\left(F^{2}+G^{2}\right)} M^{-1}
\end{array}\right] .
$$

Now, we apply the matrix $B^{-1}$ to the vector $h$ to obtain the vector $F^{\prime \prime}$. This gives the following system:

$$
\left\{\begin{array}{l}
F_{v v}=-2 F \frac{F_{v}^{2}+G_{v}^{2}}{1+2\left(F^{2}+G^{2}\right)}, \\
F_{v w}=-2 F \frac{F_{v} F_{w}+G_{v} G_{w}}{1+2\left(F^{2}+G^{2}\right)}, \\
F_{w w}=-2 F \frac{F_{w}^{2}+G_{w}^{2}}{1+2\left(F^{2}+G^{2}\right)}, \\
G_{v v}=-2 G \frac{F_{v}^{2}+G_{v}^{2}}{1+2\left(F^{2}+G^{2}\right)}, \\
G_{v w}=-2 G \frac{F_{v} F_{w}+G_{v} G_{w}}{1+2\left(F^{2}+G^{2}\right)}, \\
G_{w w}=-2 G \frac{F_{w}^{2}+G_{w}^{2}}{1+2\left(F^{2}+G^{2}\right)} .
\end{array}\right.
$$

At first look, it seems very complicated. Assume that both $F$ and $G$ are not identically zero. We deduce that

$$
\frac{F_{v v}}{F}=\frac{G_{v v}}{G} \quad \frac{F_{v w}}{F}=\frac{G_{v w}}{G} \quad \frac{F_{w w}}{F}=\frac{G_{w w}}{G},
$$

by taking ratios side by side. Let us consider the first equality. We get:

$$
F_{v v} G=G_{v v} F \text {. }
$$

Adding $F_{v} G_{v}$ to both sides, we get:

$$
F_{v v} G+F_{v} G_{v}=G_{v v} F+F_{v} G_{v}
$$

which becomes

$$
\left(F_{v} G\right)_{v}=\left(G_{v} F\right)_{v} .
$$

Integrating by parts and using the condition $G(v, 0)=F(v, 0)=0$ (same condition as in [5] and [6]), we obtain

$$
F_{v} G=G_{v} F
$$

and so (since both $F$ and $G$ must be not identically zero) one gets

$$
\frac{F_{v}}{F}=\frac{G_{v}}{G}
$$

which implies, integrating side by side, that

$$
G=F \text {. }
$$

This condition decouples the system and reduces to three the number of independent equations of our original problem of six coupled equations. Therefore, we have to solve:

$$
\left\{\begin{array}{l}
F_{v v}=-\frac{4 F F_{v}^{2}}{1+4 F^{2}}, \\
F_{v w}=-\frac{4 F F_{v}}{1+4 F^{2}}, \\
F_{w w}=-\frac{4 F F_{w}^{2}}{1+4 F^{2}} .
\end{array}\right.
$$


Let us consider the first equation:

$$
F_{v v}=-\frac{4 F F_{v}^{2}}{1+4 F^{2}} .
$$

This is the same equation (there it was in one single variable) present in [5] and [6] in the stationary case. It can be rewritten as:

$$
F_{v}=\frac{1}{\sqrt{1+4 F^{2}}}
$$

with $F(0, w)=0$ and similarly

$$
F_{w}=\frac{1}{\sqrt{1+4 F^{2}}}
$$

with $F(v, 0)=0$. But this implies that

$$
F_{v}=F_{w},
$$

which is a transport equation, whose solution is

$$
F(v, w)=F(v+w)=F_{0}(v+w)=0,
$$

because of our initial conditions. Therefore, the only solution to our system with initial conditions $G(v, 0)=F(v, 0)=G(0, w)=F(0, w)=0$ is $F=G=$ 0 , which is absurd, because we assumed that both $F \neq 0$ and $G \neq 0$. This implies that at least one between $F$ and $G$ must be zero and so that $u$ must be purely real or purely imaginary (and so real up to a constant phase shift). This implies again that $u$ must be also stationary. This concludes the proof of the theorem.

\section{Acknowledgements}

I thank my family and Victoria Ban for their constant support. I thank Prof. Yun Wang for several discussions about Section 6. I thank my supervisor Prof. Narayanaswamy Balakrishnan for his constant help and inspiring guidance. I thank the referees for their useful comments which lead to an improvement of the manuscript.

\section{References}

[1] Adachi, S., Watanabe, T.: Uniqueness of the ground state solutions of quasilinear Schrödinger equations. Nonlinear Anal. 75(2), 819-833 (2012)

[2] Berestycki, H., Lions, P.L.: Nonlinear scalar field equations I. Arch. Rat. Mech. Anal. 82, 313-346 (1983)

[3] Berestycki, H., Gallouët, T., Kavian, O.: Equationes de Champes scalaires euclidiens non linéaires dans le plan. C. R. Acad. Sci. Paris Ser. I Math. 297(5), 307310 (1983) 
[4] Colin, M.: On the local well-posedness of quasilinear Schrödinger equations in arbitrary space dimension. Commun. Partial Differ. Equ. 27, 325-354 (2002)

[5] Colin, M., Jeanjean, L.: Solutions for a quasilinear Schrödinger equation: a dual approach. Nonlinear Anal. 56, 213-226 (2004)

[6] Colin, M., Jeanjean, L., Squassina, M.: Stability and instability results for standing waves of quasi-linear Schrödinger equations. Nonlinearity 23, 13531385 (2010)

[7] Kenig, C.E., Ponce, G., Vega, L.: The Cauchy problem for quasi-linear Schrödinger equations. Invent. Math. 158, 343-388 (2004)

[8] Lange, H., Poppenberg, M., Teismann, H.: Nash-Moser methods for the solution of quasi-linear Schrödinger equations. Commun. Partial Differ. Equ. 24, 13991418 (1999)

[9] Liu, J.Q., Wang, Y.Q., Wang, Z.Q.: Soliton solutions for quasi-linear Schrödinger equations II. J. Differ. Equ. 187, 473-493 (2003)

[10] Liu, J.Q., Wang, Y.Q., Wang, Z.Q.: Solutions for quasi-linear Schrödinger equations via the Nehari method. Commun. Partial Differ. Equ. 29, 879-901 (2004)

[11] Liu, J.Q., Wang, Z.Q.: Solitons solutions for quasi-linear Schrödinger equations. Proc. Am. Math. Soc. 131, 441-448 (2003)

[12] Marzuola, J.L., Metcalfe, J., Tataru, D.: Quasilinear Schrödinger equations I: small data and quadratic interactions. Adv. Math. 231, 1151-1172 (2012)

[13] Marzuola, J.L., Metcalfe, J., Tataru, D.: Quasilinear Schrödinger equations II: small data and cubic nonlinearities. Kyoto J. Math. 54(3), 529-546 (2014)

[14] Poppenberg, M.: On the local well posedness of quasi-linear Schrödinger equations in arbitrary space dimension. J. Differ. Equ. 172, 83-115 (2001)

[15] Poppenberg, M., Schmitt, K., Zhi-Qiang, W.: On the existence of soliton solutions to quasilinear Schrödinger equations. Calc. Var. Partial Differ. Equ. 14, 329-344 (2002)

[16] Pucci, P., Servadei, R.: On weak solutions for $p$-Laplacian equations with weights. Atti Accad. Naz. Lincei Cl. Sci. Fis. Mat. Natur. Rend. Lincei Mat. Appl. 18(9), 257-267 (2007)

[17] Rudin, W.: Real and complex analysis. Int. Ser. Pure Appl. Math. 11, 232 (1986)

[18] Selvitella, A.: Uniqueness and nondegeneracy of the ground state for a quasilinear Schrödinger equation with a small parameter. Nonlinear Anal. 74(5), 17311737 (2011)

[19] Selvitella, A.: Nondegeneracy of the ground state for quasilinear Schrödinger equations. Calc. Var. PDE. 53(1-2), 349-364 (2015)

[20] Selvitella, A., Wang, Y.: Morawetz and interaction Morawetz estimates for quasilinear Schrödinger equations. J. Hyperb. Differ. Equ. 9(4), 613-639 (2012) 
[21] Taylor, M.: Partial Differential Equations III-Nonlinear Equations, 2nd edn. Applied Mathematical Sciences (2010). p. 117

Alessandro Selvitella

Department of Mathematics and Statistics

McMaster University

1280 Main Street West

Hamilton

ON L8S-4L8

Canada

e-mail: aselvite@math.mcmaster.ca

Received: 6 May 2015.

Accepted: 2 January 2016. 